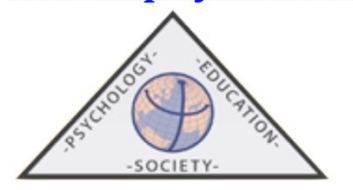

\title{
El enfoque de género en la intervención socioeducativa con mujeres: un estudio en el medio penitenciario Español*
}

\author{
Rubén BURGOS JIMÉNEZ, Bruno GARCÍA TARDÓN ${ }^{2}$, Víctor M. MARTÍN SOLBES ${ }^{3}$ \\ y Florencia POZUELO RUBIO
}

\author{
${ }^{1}$ Universidad de Granada \\ ${ }^{2}$ Universidad Camilo José Cela \\ ${ }^{3}$ Universidad de Málaga
}

${ }^{4}$ Secretaría General de Instituciones Penitenciarias

(Recibido el 8 de Mayo de 2020, Aceptado el 3 de Junio de 2020)

RESUMEN: En el presente artículo se abordan los programas de intervención socioeducativa de género desarrollados con mujeres privadas de libertad en el medio penitenciario español (en régimen ordinario y abierto). Así, a través de un cuestionario dirigido a mujeres $(\mathrm{N}=310)$ se denota que el 49\%, $(152$ casos) ha participado en algún programa socioeducativo de género durante su condena. En concreto, se resalta el programa "Ser Mujer.es" (53\% participaron en prisión y un 4\% en semilibertad) y diversos cursos y talleres educativos para promover "la igualdad de oportunidades entre hombres y mujeres" (48\% participaron en prisión y un $6 \%$ en semilibertad). A partir del análisis de los datos, se ofrece una descripción de dichos programas, analizando sus objetivos, contenidos y utilidad a través de los testimonios obtenidos en las entrevistas a mujeres $(\mathrm{N}=75)$. Por otro lado, se analiza un cuestionario dirigido a las y los profesionales que implementan dichos programas $(\mathrm{N}=66)$, para conocer la utilidad, necesidad de la perspectiva de género en la intervención, y los aspectos metodológicos y evaluativos.

En definitiva, se tiene como objetivo analizar la utilidad de los programas socioeducativos de género en el medio penitenciario, de acuerdo al proceso de reinserción de las mujeres en semilibertad. Así, se encuentra que el programa "Ser mujer.es" es valorado de utilidad por el $87 \%$ de los casos, resaltando aprendizajes sociales, comunicativos y terapéuticos e implementado por el 12,1\% de las y los profesionales. Estos programas se configuran como una herramienta vital para el proceso de reinserción femenina.

Palabras clave: Género, prisión, intervención socioeducativa, reinserción social

* El presente artículo emana del Proyecto I+D+I titulado "Procesos de reinserción socioeducativa y acompañamiento a reclusas en semilibertad" (REINAC), Referencia: EDU2016-79322-R, en el marco de la Convocatoria 2016, Programa Estatal de Investigación, Desarrollo e Innovación Orientada a los Retos de la Sociedad, financiado por el Ministerio de Economía y Competitividad, Dirección General de Investigación Científica y Técnica, Subdirección de Proyectos de Investigación 


\title{
The Gender Approach in Socio-Educational Intervention with Women:
}

A Study in the Spanish Prison Environment

\begin{abstract}
This article deals with gender socio-educational intervention programs developed with women deprived of liberty in the Spanish prison environment (in the ordinary and open regime). Thus, through a questionnaire addressed to women $(\mathrm{N}=310)$, it is denoted that $49 \%$ (152 cases) have had a gender socio-educational program during their sentence. Specifically, highlight the "Ser Mujer.es" program (53\% participate in prison and 4\% in semi-freedom) and a group of educational courses and workshops to promote "equal opportunities between men and women" (48\% participated in prison and $6 \%$ in semi-freedom). From the analysis of the data, a description of these programs is offered, analyzing their sessions, contents and usefulness through the testimonies received in the interviews with women (N $=75$ ). On the other hand, a questionnaire addressed to the professionals who implement these programs is analyzed $(\mathrm{N}=66)$, to know the usefulness, need for a gender perspective in the intervention, and the methodological and evaluative aspects.

In short, the objective is to analyze the utility of gender socio-educational programs in the prison environment, according to the process of reintegration of women in semi-freedom. Thus, the "Being a woman.es" program is found to be useful for $87 \%$ of cases, highlighting social, communicative and therapeutic learning and implemented by $12.1 \%$ of professionals. These programs are configured as a vital tool for the female reintegration process.
\end{abstract}

Keywords: Gender, prison, socio-educational intervention, social reintegration.

Correspondencia: Víctor M. Martín Solbes. Bulevar Louis Pasteur, 25, 29010 Málaga. E-mail: victorsolbes@uma.es

\section{Introducción}

Las Instituciones Penitenciarias del Estado Español se configuran como espacios que tratan de dar cumplimiento al mandato constitucional que determina la finalidad de las penas de prisión recogido en el artículo 25.2 de la Constitución Española (1978), que establece que "las penas privativas de libertad estarán orientadas hacia la reeducación y reinserción social [...] En todo caso (el condenado), tendrá derecho a un trabajo remunerado y a los beneficios correspondientes de la Seguridad Social, así como al acceso a la cultura y al desarrollo integral de su personalidad". Asimismo, los centros penitenciarios no sólo suponen entidades punitivas y funcionales del sistema, sino que se trata de espacios reeducativos que conforman un ecosistema de socialización, cultura y mejora del proceso de reinserción social del usuario (Añaños, Fernández-Sánchez y Llopis, 2013).

En este sentido, La Secretaría General de Instituciones Penitenciarias -SGIP- (2020b) se organiza en base a objetivos y fines de desarrollo de la reeducación y reinserción social, retención y custodia, y la asistencia y ayuda de los penados. Para ello, la Administración propone intervenciones, programas de tratamiento y actividades socioeducativas, culturales, deportivas y formativas enfocadas al crecimiento personal y mejora de las habilidades sociales y conductuales, así como al perfeccionamiento de competencias y a la formación laboral (SGIP, 2020c), dentro del Programa Individualizado de Tratamiento (PIT) regulado en la Instrucción 12/2006.

Sin embargo, en todo este conjunto de actividades y programas impartidos en el medio penitenciario, aún es necesario intensificar las políticas de igualdad y de perspectiva de género. La criminalidad se asocia al sexo masculino, por lo que la población femenina penada experi- 
menta una situación de exclusión y desigualdad debido a su menor presencia (Cruells, Igareda y Torrens, 2005; Añaños, 2013).

Los centros penitenciarios se configuran de acuerdo a una cultura, representaciones y estereotipos centrados en el recluso varón predominante, focalizando sus servicios de tratamiento $\mathrm{y}$ atención, y provocando una falta de adecuación y reconocimiento a las condiciones y necesidades de las mujeres internas (Herrera y Expósito, 2010; Mapelli, Herrera y Sordi, 2013). En este contexto predomina una situación de reproducción de roles, destacando el papel de mujer doméstica que participa en actividades textiles, hosteleras y de cuidado de niños, lo que limita el desarrollo de programas formativos durante su condena (Juliano, 2009). Esta exclusión se acentúa más en mujeres extranjeras debido a su situación de soledad y marginación (Añaños, 2013).

La SGIP contaba con 4.322 mujeres penadas en enero de 2020, lo que supone el 7,2\% de la población reclusa total en España. Estas mujeres comparten un periodo previo a su encarcelamiento repleto de dificultades y obstáculos sociales. Fickenscher, Lapidus, Silk-Walker y Becker (2001) manifiestan que el 79\% de las mujeres reclusas habían sufrido abuso físico, y el 67\% abuso sexual, generando problemáticas de exclusión social e inestabilidad emocional.

El ingreso en prisión de estas mujeres conlleva un proceso traumático que provoca estigmatización y rechazo social. Esta prisionalización tiene como principales consecuencias la disminución de la autoestima, dependencia emocional, problemáticas de exclusión sociolaboral, carencias afectivas, discriminación y actitudes antisociales (Herrera y Expósito, 2010).

Ante esta situación, se presenta el programa "Ser Mujer.es" realizado en las prisiones del Estado español desde 2011 con el objetivo de reducir la vulnerabilidad frente a la violencia de género de las mujeres privadas de libertad. Pretende tanto la prevención de la violencia de género como el tratamiento de aquellas que la hayan padecido. Es de carácter psicoeducativo y psicosocial, teniendo una clara orientación de género, pretendiendo que las internas aprendan a conocerse mejor, a respetarse a sí mismas, a descubrir sus capacidades y a adquirir herramientas que les permite disponer de mayores y mejores recursos. Este programa se realiza en colaboración con el Instituto de la Mujer y para la Igualdad de oportunidades del Ministerio de Igualdad (SGIP, 2011)

Por otro lado, ante las situaciones de violencia de género, numerosas investigaciones abordan la necesidad de implementar programas formativos con las personas agresoras, relacionadas con el análisis de la violencia ejercida por hombres (Rodríguez-Espartal y López-Zafra, 2013; Sordi, 2016); mientras que otros estudios, focalizan los programas dirigidos hacia las mujeres víctimas de violencia (Fontanil, Alcedo, Fernández y Ezama, 2013). Por ello, la SGIP desarrolla programas y actuaciones de intervención dirigidos tanto a los agresores condenados por violencia de género (Programa PRIA-MA) como a las mujeres del medio para la prevención y el tratamiento de la violencia de género, abordando las situaciones de abuso o maltrato en las relaciones de pareja a través de intervenciones individuales y grupales.

En cuanto a las y los profesionales que llevan a cabo estos programas, son fundamentalmente los especialistas en psicología, dado el carácter terapéutico de los programas, actuando junto a otros profesionales del medio como educadores y educadoras o trabajadores y trabajadoras sociales.

En definitiva, se pretende aproximar las experiencias de las mujeres penadas relacionadas con la implementación de los programas y las percepciones de las y los profesionales que los realizan, respecto a cuestiones relacionadas con la oportunidad de la acción, la perspectiva de género, los abordajes metodológicos y el éxito. 


\section{Método}

El diseño metodológico de este trabajo forma parte de la propuesta en el proyecto I+D+I "Procesos de Reinserción Socioeducativa y Acompañamiento a Reclusas en Semilibertad" (REINAC) Ref.EDU2016-79322-R. Es descriptivo e interpretativo, siendo un diseño multimétodo, en el que se combina tanto métodos cualitativos como cuantitativos, en función de la coherencia, amplitud y multidimensionalidad del problema de estudio. Se tiene como objetivo principal de investigación, analizar los programas de género en el medio penitenciario y su repercusión en los procesos personales-formativos para la preparación de la vida en libertad desde la perspectiva de las mujeres y la de las y los profesionales.

La población objeto de estudio son las mujeres en fase de semilibertad, independientemente de su situación social, laboral o de consumo en los distintos Centros de Inserción Social y Secciones Abiertas de los centros penitenciarios seleccionados del Estado Español, a través de una muestra de 310 mujeres mediante un muestreo bietápico, siendo la primera fase un muestreo estratificado previo, cuyo requisito para construir los estratos será la Comunidad Autónoma en la que se cumple la condena y, como segunda etapa, será un muestreo aleatorio simple de las unidades de cada estrato. Para tener otro punto de referencia sobre esta realidad de estudio, se analizan las percepciones de las y los profesionales que trabajan con este colectivo, consiguiendo una muestra de 66 profesionales del medio penitenciario.

En cuanto a los instrumentos de investigación, se utilizan cuestionarios destinados tanto a mujeres reclusas $(\mathrm{N}=310)$ de 115 preguntas, como profesionales del medio $(\mathrm{N}=66)$ de 100 preguntas, además de entrevistas semiestructuradas a internas $(\mathrm{N}=75)$ con 68 preguntas. Los instrumentos se han administrado voluntariamente, tras obtener la autorización y el consentimiento informado.

Para la obtención de la información, se seleccionan las preguntas e ítems de los cuestionarios y de la entrevista que responden a los objetivos de la investigación, con las que se indagará en las categorías delimitadas del estudio global. En concreto, se analizan las preguntas 66, 67 y 68 del cuestionario a mujeres, la pregunta 40 de las entrevistas de las internas y, por último, las preguntas 18, 26, 30 y 31 del cuestionario dirigido a profesionales del medio. El número de hojas que han resultado de la transcripción de las entrevistas han sido treinta, de las que las preguntas referenciadas, ocupan un total de quince.

Para analizar la información cuantitativa obtenida se utilizó el programa SPSS (versión 23 y 24); mientras que para la información cualitativa (entrevistas y preguntas abiertas de los cuestionarios) se usó el programa Nvivo (versión 12) junto al análisis de contenido (cualitativo).

\section{Resultados}

En primer lugar, los instrumentos abordan la realización y valoración de los programas socioeducativos específicos en perspectiva de género dirigidos a mujeres en el medio penitenciario (tabla 1 y 2). Así, se observa que el 46,1\% de las reclusas que cumplimentaron el cuestionario $(\mathrm{N}=310)$ han participado en este tipo de programas durante el cumplimiento de su condena en régimen ordinario (prisión), mientras que en régimen abierto (semilibertad) este porcentaje disminuye al 11,9\%. 
Tabla 1. Programas socioeducativos y de género realizados por las internas $(\mathrm{CM}, 66)$

\begin{tabular}{|c|c|c|c|c|c|c|c|}
\hline & \multicolumn{7}{|c|}{ Programas socioeducativos y de género realizados } \\
\hline & $\begin{array}{c}\text { Régimen } \\
\text { Ordinario } \\
\text { (Prisión) } \\
\text { N }\end{array}$ & $\begin{array}{c}\text { Régimen } \\
\text { Ordinario } \\
\text { (Prisión) } \\
\%\end{array}$ & $\begin{array}{r}\text { Régime } \\
\text { (semil }\end{array}$ & $\begin{array}{l}\text { n abierto } \\
\text { ibertad) } \\
\mathbf{N}\end{array}$ & $\begin{array}{c}\text { Régimen abierto } \\
\text { (semilibertad) } \\
\%\end{array}$ & $\begin{array}{l}\text { Totales } \\
\quad \mathbf{N}\end{array}$ & $\begin{array}{c}\text { Totales } \\
\%\end{array}$ \\
\hline $\begin{array}{l}\text { Mujeres que han realizado } \\
\text { programas socioeducativos } \\
\text { y de género }\end{array}$ & 143 & $46,1 \%$ & B & 37 & $11,9 \%$ & 310 & $100 \%$ \\
\hline $\begin{array}{l}\text { Programa } \\
\text { "Ser Mujer.es" }\end{array}$ & 80 & $52,6 \%$ & V & 6 & $3,9 \%$ & 152 & $100 \%$ \\
\hline $\begin{array}{l}\text { Programa de "Promoción } \\
\text { de la igualdad" }\end{array}$ & 74 & $48,7 \%$ & & 9 & $5,9 \%$ & 152 & $100 \%$ \\
\hline Otros programas & 7 & $4,6 \%$ & & 7 & $4,6 \%$ & 152 & $100 \%$ \\
\hline
\end{tabular}

Fuente: elaboración propia a partir del cuestionario de mujeres del proyecto EDU2016-79322-R

Tabla 2. Valoración de la utilidad de los programas socioeducativos $y$ de género realizados por mujeres $(\mathrm{CM}, 66)$

\begin{tabular}{|c|c|c|c|c|c|c|c|c|}
\hline & \multicolumn{8}{|c|}{ Valoración sobre la utilidad de los programas realizados } \\
\hline & $\begin{array}{c}\text { Régimen } \\
\text { Ordinario } \\
\mathbf{N}\end{array}$ & $\begin{array}{c}\text { Régimen } \\
\text { Ordinario } \\
\%\end{array}$ & $\begin{array}{c}\text { Totales } \\
\text { Régimen } \\
\text { Ordinario } \\
\text { N }\end{array}$ & $\begin{array}{c}\text { Totales } \\
\text { Régimen } \\
\text { Ordinario } \\
\%\end{array}$ & $\begin{array}{c}\text { Régimen } \\
\text { abierto } \\
\mathbf{N}\end{array}$ & $\begin{array}{c}\text { Régimen } \\
\text { abierto } \\
\%\end{array}$ & $\begin{array}{c}\text { Totales } \\
\text { Régimen } \\
\text { abierto } \\
\text { N }\end{array}$ & $\begin{array}{c}\text { Totales } \\
\text { Régimen } \\
\text { abierto } \\
\%\end{array}$ \\
\hline $\begin{array}{l}\text { Programa } \\
\text { "Ser Mujer.es" }\end{array}$ & 70 & $87,5 \%$ & 80 & $100 \%$ & 6 & $100 \%$ & 6 & $100 \%$ \\
\hline $\begin{array}{l}\text { Programa de } \\
\text { "Promoción de } \\
\text { la igualdad" }\end{array}$ & 63 & $8 \quad 85,1 \%$ & 74 & $100 \%$ & 9 & $100 \%$ & 9 & $100 \%$ \\
\hline $\begin{array}{l}\text { Otros } \\
\text { programas }\end{array}$ & 56 & $90,3 \%$ & 62 & $100 \%$ & 15 & $88,2 \%$ & 17 & $100 \%$ \\
\hline
\end{tabular}

Fuente: elaboración propia a partir del cuestionario de mujeres del proyecto EDU2016-79322-R

\section{Programa "Ser mujer.es"}

En el cuestionario de mujeres se destaca la participación en el programa "Ser Mujer.es" en el $52,6 \%$ de las participantes durante su estancia en prisión y en el 3,9\% durante su fase actual de semilibertad. Esta intervención es valorada positivamente por el $87,5 \%$ de las mujeres que lo realizaron en prisión y por el $100 \%$ en semilibertad.

Asimismo, en las entrevistas a reclusas $(\mathrm{N}=75)$ este programa se describe como un espacio destinado al reconocimiento, motivación y empoderamiento para las mujeres. En concreto, el $60 \%$ de ellas, mencionan la realización del programa durante su condena.

Respecto a los beneficios que ha supuesto la participación en este tipo de actuación para su proceso de reinserción, durante la entrevista, el $40 \%$ de las internas que han participado refieren 
aprendizajes de motivación y superación personal, dando lugar a un incremento de su autoestima y habilidades sociales. El 30\% menciona la identificación y valoración de sus vínculos y relaciones sociales para la prevención de situaciones de violencia. Por último, el 25\% destaca la necesidad de implementar más contenidos y actividades durante su realización.

Con todo, el 44\% de las internas que han participado en el programa "Ser mujer.es" lo recomiendan como una actuación ideal durante el cumplimiento de su condena para el desarrollo de su proceso de reinserción y reeducación personal.

\section{Cursos y talleres dirigidos a "promover la igualdad de oportunidades entre hombres y mujeres"}

En segundo lugar, se encuentran los programas de "Igualdad de oportunidades", enfocados a disminuir la desigualdad entre hombres y mujeres, educando en derechos e identidad de género. Estos programas son identificados en el cuestionario de mujeres $(\mathrm{N}=310)$ con una participación del 48,7\% durante su estancia en prisión y del 5,9\% en semilibertad.

En este sentido, el $85,1 \%$ de las internas que han realizado estos programas en prisión lo valora de gran utilidad, mientras que el 100\% que están en semilibertad lo valoran de la misma forma. La utilidad de estos programas también se refleja en las entrevistas realizadas, que indican un porcentaje de participación del $40 \%$ en estos programas.

En cuanto a los aprendizajes significativos de estos programas, el 37,5\% de las entrevistadas valoran la adquisición de autonomía, superación y autogestión personal. El 25\% refiere aprendizajes basados en apoyo emocional, resaltando la escucha activa, comprensión y comunicación asertiva con las y los profesionales, como la identificación y gestión de las situaciones y relaciones de abuso y violencia.

El 12,5\% de las entrevistas respondieron negativamente acerca de la utilidad de los cursos: "nada. La verdad que no...pasar la mañana haciendo algo allí dentro, la verdad" (204EAA). Por otro lado, también deben considerarse los motivos por los que se abandonan este tipo de cursos, tal y como señala la participante 301ENA "no lo terminé porque me puse en el curso de la Caixa de cocina para incorporarme a trabajar".

Con todo, en el cuestionario de mujeres identifica una demanda de participación en este programa del 17\% que no lo han realizado, seguido de un 44\% de recomendaciones positivas de las mujeres que sí participaron.

\section{Otros tipos de programas socioeducativos}

Por último, en el cuestionario de mujeres $(\mathrm{N}=310)$ se menciona la participación en otro tipo de programas, destacando la realización de actividades socioculturales, definidas por la SGIP (2020c) como acciones de ocio y cultura destinadas al incremento de la creatividad. Se observa un porcentaje de participación del 40,7\% de las participantes en prisión y el 11,1\% en semilibertad. Estos programas se describen como acciones formativas y favorables en el desarrollo de su proceso de reinserción en el 90,3\% de las reclusas que han participado en prisión y el 88,2\% en semilibertad.

En especial, se muestran actividades de teatro, pintura, manualidades y costura. Asimismo, durante la entrevista $(\mathrm{N}=75)$ se menciona la realización de dichos programas en el $20 \%$ de las participantes, destacando la actividad de costura como espacios de reunión y socialización terapéutica para dicho colectivo. 
Por otro lado, se incide en las aportaciones de estos cursos para su futuro laboral: "así aprendes y te ayuda porque si tienes recursos e información que has aprendido de allí pues en la calle me puede servir" (297ENA). La participante 197ENA también resalta los beneficios laborales de estas actividades: "trabajar en equipo, en regirte a las normas que si estás en un trabajo tienes que ocupar tu lugar, no puedes ir por encima de nadie y si tienes un grupo tienes que respetar, todas esas cosas". Estas participantes también destacan el deporte en sus reflexiones.

\section{Percepción profesional de los programas de género en el medio penitenciario}

Esta investigación también pretende conocer la percepción que, desde el ámbito profesional, se tiene sobre la implementación de estos programas de intervención socioeducativa. En este sentido, mediante el cuestionario dirigido a profesionales del medio $(\mathrm{N}=66)$ se destacan los siguientes resultados:

En primer lugar, se describe la situación académica personal y profesional, encontrando profesionales de la psicología en el 33\% de los casos, trabajo social, en el 19\% y educadoras y educadores en el 15\%. Estas profesiones se constituyen como perfiles relacionados con el ámbito de la psicología, la educación y formación.

En cuanto a su situación laboral, el $47 \%$ de los/as profesionales son funcionarios y funcionarias de la Institución Penitenciaria, casi el 41\% se trata de trabajadores y trabajadoras de entidades colaboradoras y el 1,5\% personas relacionadas con una actividad de voluntariado.

Respecto a la tipología de programas socioeducativos realizados por el personal del medio penitenciario, el 12\% se encarga de la implementación de programas de género (tabla 3), siendo el tercer tipo de programas realizados junto a los programas laborales, en el 22,7\% de los casos, y los programas de acompañamiento en el 16,7\%.

Tabla 3. Tipología de programas socioeducativos realizados por los y las profesionales $(C P, 18)$

\begin{tabular}{lcc}
\hline & \multicolumn{2}{c}{ Tipología de programa realizado } \\
\cline { 2 - 3 } & $\mathbf{N}$ & $\mathbf{\%}$ \\
\hline Programas laborales & 15 & $22,7 \%$ \\
Programas de acompañamiento & 11 & $16,7 \%$ \\
Programas de género & 8 & $12,1 \%$ \\
Programas de tratamiento de drogodependencias & 7 & $10,6 \%$ \\
Otro tipo de programas & 16 & $24,3 \%$ \\
En blanco & 9 & $13,6 \%$ \\
TOTAL & 66 & $100 \%$ \\
\hline
\end{tabular}

Fuente: Elaboración propia a partir del cuestionario de profesionales del proyecto EDU2016-79322-R

Sin embargo, a pesar de encontrar un porcentaje bajo (12\%) en la implementación de programas de género, el $80 \%$ de los programas realizados por estos profesionales, de un modo u otro, ponen en valor la perspectiva de género. Además, el 63\% de ellos, que realizan actuaciones 
socioeducativas en el medio penitenciario, considera que dichos programas cumplen con una alta adaptación a la situación sociolaboral y personal de las mujeres.

Respecto a las opciones de los 8 profesionales encargados de la realización del programa "Ser Mujer.es" y de "igualdad de oportunidades", se resalta la focalización de objetivos de atención y formación en habilidades sociales, comunicativas y personales (25\%), disminuir las desigualdades de género en el medio penitenciario (25\%) y la preparación para la vida en libertad $(12,5 \%)$.

Para ello, estos programas se configuran mediante métodos de organización grupal (25\%), asambleas terapéuticas de expertos y expertas $(25 \%)$ y visitas y salidas al exterior $(12,5 \%)$. Estas técnicas se presentan como la manera más idónea de trabajo, observando un porcentaje de participación muy alto en el $87,5 \%$ de los casos y con una finalización exitosa en el 62,5\%. Se configura como una herramienta vital para la estancia de las mujeres penadas, consiguiendo una satisfacción profesional respecto a la consecución de los objetivos propuestos en el $50 \%$.

En cuanto a la evaluación de estos programas, el 62,5\% indican un proceso de evaluación inicial diagnóstico para la adaptación y adecuación de las actuaciones. El 50\% realizan evaluaciones durante la implementación, valorando la consecución de los objetivos propuestos. Por último, el 62,5\% muestra una evaluación final para valorar el éxito de sus intervenciones, sin embargo, sólo 37\% realiza evaluaciones globales "ex-post", para el perfeccionamiento de futuras aplicaciones.

\section{Discusión}

El medio penitenciario se establece como un espacio reeducativo y formador, más allá de su función punitiva y sancionadora (Añaños et al., 2013), contando con multitud de intervenciones socioeducativas para la mejora de la reinserción de la mujer. Sin embargo, estas acciones se ven reducidas en la fase de semilibertad, debido a las características personales (Loiranz y Andrés-Pueyo, 2017) y de dicho régimen, denotando una bajada en la realización de las intervenciones del $46,1 \%$ al $11,9 \%$, lo que dificulta la formación y acompañamiento profesional.

Frente a las carencias presentadas en la política de intervención con perspectiva de género en el medio penitenciario (Cruells et al., 2005) el programa "Ser mujer.es" se presenta como una iniciativa de atención integral y ajuste a las necesidades y vulnerabilidad de estos perfiles (SGIP, 2009). Este programa es valorado positivamente por el 87,5\% de las mujeres en prisión y el $100 \%$ en semilibertad. Esta valoración es señalada también en el Documento Penitenciario número 21 de la evaluación realizada de este programa entre los años 2010 y 2016, "el efecto positivo sobre las redes sociales y familiares, particularmente sobre los hijos. El cambio y la voluntad de cambio es una de las cuestiones que mejor explica la decisión de abandonar el delito. El Programa lo propicia" (Viedma y Del Val, 2019, p. 71).

Supone mejoras terapéuticas y educativas a nivel de habilidades sociales, comunicativas y afectivas, ante las dificultades de exclusión y estigmatización del colectivo (Herrera y Expósito, 2010), además de la creación de un espacio exclusivo para la atención de las mujeres y alejado de desigualdades y diferencias de género en el trato profesional (Añaños, 2013). Sin embargo, el $25 \%$ de las entrevistadas demandan mayor variedad de contenidos en dicho programa.

Por otra parte, los programas de "Igualdad de oportunidades" se centran en el abordaje de situaciones y/o relaciones de abuso para la prevención y empoderamiento de las mujeres hayan o no sufrido malos tratos (Fontanil, Alcedo, Fernández y Ezama, 2013; SGIP, 2011;). Al igual que el programa "Ser mujer.es", esta iniciativa es apreciada de utilidad y significación en el $85,1 \%$ 
de las participantes en régimen cerrado y el 100\% en régimen abierto. Se resaltan aprendizajes emocionales, denotando problemáticas y carencias de inestabilidad, dependencia emocional y exclusión social debido a su historia de vida (Loiranz y Andrés-Pueyo, 2017).

Las actividades socioculturales y deportivas, se contemplan como programas interesantes, que requieren un estudio y análisis pormenorizado ya que corresponden a los objetivos previstos en el artículo 25.2 de la Constitución (Castillo, García-Tardón y Pardo, 2019). La actividad de costura refleja una situación de reproducción de roles interiorizada, ejercida a través de un rol femenino doméstico y textil (Juliano, 2009).

En cuanto a las miradas de las y los profesionales que acometen las acciones socioeducativas a través de estos programas, se destaca que los encargados de cumplimentar los programas socioeducativos son mayoritariamente psicólogas y psicólogos, trabajadoras y trabajadores sociales y educadoras y educadores.

Sin embargo, se considera a las educadoras y educadores sociales como el perfil profesional adecuado en la realización de la acción socioeducativa, aunque como se recoge en diversos estudios (Gil-Cantero, 2010; Martín-Solbes, Vila y de Oña, 2013; Martín-Solbes y Vila, 2016; Valderrama, 2013), la educación social es una actividad profesional desregularizada en la Institución Penitenciaria, lo que evidencia, por un lado, la escasa competencia profesional para asumir acciones socioeducativas, más allá de la buena voluntad y, por otro, la inexistente voluntad política para solucionar esta anomalía central en el desarrollo de los programas y de toda la acción socioeducativa desarrollada (Martín-Solbes y Vila, 2016; Valderrama, 2013); además, el acceso a los puestos de educadores y educadoras se realiza a través de un curso de capacitación, independientemente de la formación inicial y teniendo en cuenta la antigüedad en el puesto de funcionariado de vigilancia, lo que supone una grave anomalía en el desarrollo profesional de los agentes socioeducativos.

Respecto a los programas "Ser Mujer.es" y "Promoción de la igualdad", las y los profesionales que lo realizan trabajan objetivos relacionados con la mejora de las habilidades sociales y personales de las mujeres para la preparación de su vida en libertad y la disminución de las desigualdades de género en el medio penitenciario. Para ello, se realiza una evaluación inicial destinada a la adaptación y adecuación del programa a la situación social, personal y educativa de la mujer.

Por último, enfatizar que desde la promulgación de la Ley 1/1979, de 26 de septiembre, General Penitenciaria, que marca las actuaciones en la Administración Penitenciaria hacia la reeducación y reinserción social de acuerdo al artículo 25.2 de la Constitución Española de 1978, se han dado importantes pasos en la atención especial de las mujeres privadas de libertad. Aun así, la situación de desigualdad de género, sigue estando muy presente en este colectivo. Los resultados de este estudio sirven para reflexionar sobre la necesidad de incrementar e incidir en programas formativos y socioeducativos con enfoque de género, tanto en la situación de privación de libertad como de semilibertad que facilite el empoderamiento de las mujeres y la adecuación progresiva hacia la vida en libertad, minimizando la desigualdad social y de género existente todavía dentro del medio penitenciario.

\section{Referencias}

Añaños, F. (2013). Formación educativa previa ante las discriminaciones: las mujeres reclusas en España Prior Education and Discrimination: Women in Spanish Prisons. Revista de Educación, 360, 91-118. 
Añaños, F, Fernández-Sánchez, M y Llopis, J. (2013). Aproximación a los contextos en prisión: una perspectiva socioeducativa. Pedagogía social: revista interuniversitaria, (22), 2-16.

Constitución Española (1978). Publicada en BOE de 29 de diciembre de 1978.

Castillo-Algarra, J., García-Tardón, B., y Pardo, R. (2019). Sport in Spanish prisons: Towards the Third Degree or the Third Half? (Deporte en las prisiones españolas ¿hacia el tercer grado o el tercer tiempo?). Cultura_Ciencia_Deporte, 14(40), 5-13.

Cruells, M., Igareda, N., y Torrens, M. (2005). La inclusión de la perspectiva de género en los marcos penales. En Cruells e Igareda (eds.) Mujeres, Integración y Prisión, pp. 7981. Barcelona: SURT.

Dirección General de Instituciones Penitenciarias (2006). Instrucción 12-2006. Programación, evaluación e incentivación de actividades y programas de tratamiento.

Fickenscher, A., Lapidus, J., Silk-Walker, P., y Becker, T. (2001). Women behind Bars: Health Needs of Inmates in a County Jail. Public Health Reports, 116(3), 191-196.

Fontanil, Y.; Alcedo, Ma.A.; Fernández, R. y Ezama, E. (2013). Mujeres en prisión: un estudio sobre la prevalencia del maltrato. Revista Española de Sociología, 20, 21-38.

Gil-Cantero, F. (2010). La acción pedagógica en las prisiones. Posibilidades y límites. Revista Española de Pedagogía, 245, 49-64.

Herrera, C., y Expósito, F. (2010). Una vida entre rejas: Aspectos psicosociales de la encarcelación y diferencias de género. Psychosocial Intervention, 19(3), 235-241.

Juliano, D. (2009). Delito y pecado. La transgresión en femenino. Política y sociedad, 46(1), 79-95.

Loiranz, I., y Andrés-Pueyo, A. (2017). Victimización en la pareja como factor de riesgo en mujeres en prisión. Revista Criminalidad, 59(3), 153-162.

Mapelli, B., Herrera, M., y Sordi, B. (2013). La exclusión de las excluidas. ¿Atiende el sistema penitenciario a la necesidad de género?: una visión andaluza. Estudios penales y criminológicos, 33, 59-95.

Martín-Solbes, V. M., Vila, E. y De Oña, J. (2013). La investigación educativa en el ámbito de las Instituciones Penitenciarias. Panorámica, desafíos y propuestas. Revista de Educación, 360, 16-35.

Martín-Solbes, V. y Vila, E. (2016). Si nada cambia, todo continúa igual. La educación social y sus ausencias en el ámbito penitenciario. Revista de Educación Social (RES), 22, 11-27.

Secretaría General de Instituciones Penitenciarias (2011). Programa de prevención de violencia de género para las mujeres en Centros Penitenciarios: Ser mujer.es Recuperado el 1 de marzo de 2020 de: http://www.institucionpenitenciaria.es/web/export/sites/default/datos/ descargables/publicaciones/Documento_Penitenciario_9_Ser_Mujer_profesionales.pdf

Secretaría General de Instituciones Penitenciarias. (2019). Evaluación de la eficacia de un programa de tratamiento para el empoderamiento de mujeres en prisión. Programa Sermujer.es. Recuperado el 1 de marzo de 2020 de: http://www.institucionpenitenciaria.es/ web/export/sites/default/datos/descargables/publicaciones/Documento_Penitenciario_21. Evaluación de la eficacia de un programa de tratamiento para el empoderamiento de mujeres en prision. Programa Sermujer,es. pdf 
Secretaría General de Instituciones Penitenciarias. (2020a). Estadística penitenciaria: distribución de la población reclusa por sexo. Recuperado el 1 de marzo de 2020

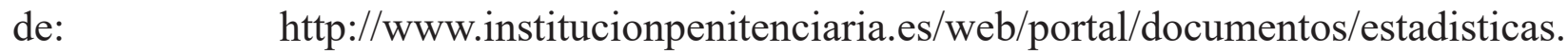
$\mathrm{htm} l \mathrm{r}=\mathrm{m} \& \mathrm{adm}=\mathrm{TES} \& \mathrm{am}=2020 \& \mathrm{~mm}=1 \& \mathrm{tm}=\mathrm{GENE} \& \mathrm{tm} 2=\mathrm{GENE}$

Secretaría General de Instituciones Penitenciarias. (2020b). La Administración Penitenciaria: fin y principios. Recuperado el 1 de marzo de 2020 de: http://www.institucionpenitenciaria.es/ web/portal/administracionPenitenciaria/finPrincipios.html

Secretaría General de Instituciones Penitenciarias. (2020c). Reeducación y reinserción social. Recuperado el 13 de marzo de 2020 de: http:/www.institucionpenitenciaria.es/web/portal/ Reeducacion/programasOcioyCultura.html

Secretearía General de Instituciones Penitenciarias. (2020d). Programas específicos: Programa PRIA-MA. Recuperado el 22 de marzo de 2020 de: http://www.institucionpenitenciaria.es/ web/portal/PenasyMedidasAlternativas/programas/priama.html

Sordi, B. (2016). Programas para agresores de violencia de género en prisión: ¿De qué evidencia disponemos? Revista Española de Investigación Criminológica, 13 (6), 1-30.

Valderrama, P. (2013). Cárcel, poder y conflicto. La micropolítica de la función reeducadora. Málaga: Servicio de Publicaciones de la Universidad de Málaga.

Viedma y Del Val, (2019). Evaluación de la eficacia de un programa de tratamiento para el empoderamiento de las mujeres en prisión. (Programa Sermujer.es). Madrid: Ministerio de interior. 